\title{
New analysis software for Viking Lander meteorological data
}

\author{
O. Kemppinen ${ }^{1,2}$, J. E. Tillman ${ }^{3}$, W. Schmidt ${ }^{1}$, and A.-M. Harri ${ }^{1}$ \\ ${ }^{1}$ Earth Observation, Finnish Meteorological Institute, Helsinki, Finland \\ ${ }^{2}$ School of Science, Aalto University, Espoo, Finland \\ ${ }^{3}$ Department of Atmospheric Sciences, University of Washington, Seattle, Washington, USA
}

Correspondence to: O. Kemppinen (osku.kemppinen@fmi.fi)

Received: 23 August 2012 - Published in Geosci. Instrum. Method. Data Syst. Discuss.: 5 October 2012 Revised: 4 January 2013 - Accepted: 16 January 2013 - Published: 6 February 2013

\begin{abstract}
We have developed a set of tools that enable us to process Viking Lander meteorological data beyond what has been previously publicly available. Besides providing data for new periods of time, the existing data periods have been augmented by enhancing the data resolution significantly. This was accomplished by first transferring the original Prime computer version of the data analysis software to a standard Linux platform, and then by modifying the software to be able to process the data despite irregularities in the original raw data and reverse engineering various parameter files. In addition to this, the processing pipeline has been streamlined, making processing the data faster and easier.

As a case example of new data, freshly processed Viking Lander 1 and 2 temperature records are described and briefly analyzed in ways that have not been previously possible due to the lack of data.
\end{abstract}

\section{Introduction}

The Viking Mission launched in 1975 was the first successful lander mission to Mars, arriving on the Martian orbit in the summertime 1976. The Mission had a versatile set of scientific objectives. Primarily, the Viking Mission was aiming at the investigations of the current or past existence of life on Mars, but other experiments were also designed, including meteorological and seismological measurements and experiments on the composition of the atmosphere (Williams, 2011; Chamberlain et al., 1976; Soffen, 1977).

The mission consisted of two separate unmanned spacecraft, Viking 1 and 2. Each spacecraft further consisted of an orbiter and a lander. The Viking landers 1 and 2 (VL1 and VL2) were each expected to be operational for 90 Mars solar days (sols), later changed to 60 sols for VL2 (Soffen, 1977; Snyder, 1977). The Viking Mission was a tremendous success in all aspects of planetary science. Especially important from the atmospheric sciences point of view was the provision of a record of atmospheric observations on two surface sites during a period of several Martian years.

After Viking there was a hiatus of about two decades before the Martian in situ exploration was successfully resumed. The importance of the Viking Mission is underlined by the fact that all the Martian exploration missions after Viking are referring to the Viking data records. This is especially the case for all the landed Mars missions with atmospheric observations, e.g. Mars Pathfinder, Phoenix, Mars Science Laboratory (Linkin et al., 1998; Harri et al., 1998; Golombek et al., 1999; Savijärvi et al., 2004, 2005; Shotwell, 2005; Taylor et al., 2008, 2010).

The Viking landers operated significantly longer than expected: VL1 was operational for 2245 sols and VL2 was operational for 1281 sols. After about sol 1000 of the VL1 mission the responsibility of storing the data was transferred from Jet Propulsion Laboratory (JPL) to James E. Tillman and his University of Washington Viking Computer Facility (UW VCF) staff, who made it possible for the Viking program to provide yet unmatched amount of long-term meteorological data (Tillman et al., 1988). The earliest possible year for any lander to surpass the extent of the Viking direct meteorological data is 2018, when Mars Science Laboratory may have been on Mars for over 2245 sols. It is worth noting that Mars Exploration Rover Opportunity has been operational on the surface of Mars for over 2245 sols, and that it carries a Mini-TES instrument capable of making some indirect measurements of meteorology (Smith et al., 2006). 
Each Viking Lander had instruments for several fields of science, such as geology, imaging and meteorology. The meteorology instruments included a pressure sensor, a temperature sensor and wind sensors for velocity and direction measurements. The complete pressure data of both landers has been published in the Planetary Data System (PDS), but the temperature and wind data found in the PDS is severely incomplete. For VL1, the published temperature and wind data covers the first 350 sols of the 2245 -sol mission. VL2 mission is covered completely (1281 sols). However, for every sol only 25 temperature and wind data point averages are provided, whereas the full data amount is between 150 and 2000 measurements per sol. Therefore, in addition to the large missing portions of the VL1 data, the resolution of the published temperature and wind data is severely limited.

When the support at the UW for the original Viking team ended in 2004, Finnish Meteorological Institute (FMI) as a longstanding cooperation partner in Mars meteorological sciences was contacted with the request to continue the work on the data and to upgrade the original data analysis software from FORTRAN IV format of the Prime computer system to one that works in a standard Linux environment. At FMI, the original FORTRAN IV code was adapted to the Intel Fortran 90 compiler, the C-routine package was completely rewritten for the Linux environment and the Command Procedure Language (CPL) process control scripts were translated into Perl scripts. The original software is briefly described in Sect. 2 and the current processing pipeline is described in Sect. 3.

In addition to the platform changes, the software has been significantly modified to be able to analyze data that has been unavailable until now. The first results are summarized in Sect. 4.

This is the first article describing the preliminary new data produced with the new version of the software. The main purpose is to give an overview of the data processing software and describe the current version of the data. A more complete overview and in-depth analysis of the data will appear in subsequent articles.

The data discussed in this article consists only of the temperature measurements. The atmospheric pressure data were engineering measurements, serving both entry and landing needs, so they were processed separately from meteorology. In addition, the wind data validity is still being verified. For VL1, the main issue is that the software changes made to account for a malfunction of the quadrant sensor heater and a failure of one wind sensor cause singularities for wind speed. For VL2, the processing package produces non-outlier noon wind velocities of up to $80 \mathrm{~m} \mathrm{~s}^{-1}$ near sol 700 , which forces us to consider that there might be a problem with processing even in the intact wind instrument data.

\section{The original Prime-based analysis software (OPS)}

Except for atmospheric pressure, the original software was completed in 1975 and was written for Universal Automatic Computer (UNIVAC) 1108 computer system by Martin Marietta corporation, for the purpose of analyzing Viking lander meteorological data (Buehler, 1974b,a). In 1976 it was ported to FORTRAN IV to be used with the UW Prime 400 computer. The development continued at VCF, where it was used for all routine data analysis of the extended mission data products and for special data analysis in connection with the preparation of scientific publications (Tillman et al., 1988).

Since the Prime 400 version, the software has had four core software packages, processed linearly in order. Most of these packages were written in FORTRAN IV, but a bit manipulation package was written in $\mathrm{C}$ and adapted to the operating system environment. The core software packages were controlled by Command Procedure Language process control scripts, feeding configuration parameters from various databases together with the original or pre-processed data sets to the FORTRAN IV software. Many of these configuration parameters were based on mission operation information logged manually during the mission. When the responsibility for the data processing was transferred to the UW, the new crew started to use calibration information collected during the first months of instrument operation on the Martian surface and during additional wind tunnel experiments on the ground. These values deviated somewhat from the parameters used by the JPL system (Tillman et al., 1988).

\section{Updated Linux-based Viking analysis toolbox (ULT)}

ULT is a new tool for Viking lander meteorological data analysis. It is in essence an update of the OPS that has been modified to cope with several irregularities in the raw data that cause the original version to crash. ULT runs in a local FMI Linux cluster, but works in any Linux environment, such as a personal computer or a cloud. Furthermore, the software was streamlined in a way that processing the data for the whole mission does not require non-trivial human interaction. In addition to modifying the original software pieces to run automatically, the streamlining was accomplished by creating a generator script for essential <names $>$ parameter files, described below in more detail. In the Linux version, we have preserved the software and data file naming conventions of the Prime version of the software.

In the text below, software packages and their subcomponents are written in capital letters, for example SANMET. File type names are enclosed in symbols $<$ and $>$, for example $<$ MFILE $>$, to make it explicit which terms correspond to software packages and which terms correspond to data files.

The original raw data stream from both of the spacecraft was stored in Intermediate Data Record tapes. These tapes 
Table 1. Meteorology frame extraction (DECSET).

\begin{tabular}{ll}
\hline Name & Description \\
\hline SETUP & Reads in parameters, opens files, reads their contents \\
EXPAND & Discards overhead bytes from the data \\
NXFRAM & Reads meteorological data frames by searching for synch words from the data \\
METOUT & Combines the read frames \\
OUTPUT & Writes the output \\
\hline
\end{tabular}

Table 2. Data time ordering (PREFEP).

\begin{tabular}{ll}
\hline Name & Description \\
\hline INIT & Reads in parameters and initializes variables \\
READIN & Reads the data frames \\
TIMESORT & Sorts the data frames according to epoch and GCSC times \\
MRKSOL & Matches the data to known LSEQ cards to create data records from the frames and data modules from the records \\
FITSOL & Varies epoch and GCSC times to see if additional modules can still be found from the leftover data \\
OUT & Outputs the data \\
\hline
\end{tabular}

were converted to Master Data Record (MDR) tapes with Decommutation and Decalibration Set Software (DECSET) in JPL and later in VCF (Tillman et al., 1988).

The software information flow is summarized in Fig. 1. Below, each part of the program is described in more detail. The naming convention of the sub-parts of the programs are loosely based on the actual subroutines. As the objective is to describe the general structure of the programs, in tables below some of the helper routines are omitted or merged to the main subparts for the sake of clarity. The program runs the subroutines linearly in the order shown in the tables.

First, DECSET extracts complete frames from the unformatted bit stream contained in MDR files by searching for frame synchronization patterns. Next, it discards the frames which are not meteorological or desired, and finally it outputs the meteorological frames in a format understood by the next program, Pre-Front End Processor (PREFEP). This format is called $<$ FMT 7 metout $>$. DECSET general structure and the individual subparts of the program are briefly described in Table 1.

PREFEP reads meteorology frames from $<$ FMT 7 metout $>$ files and checks frames to see if they fit in an appropriate module and record times that are provided either manually by the user or, as done in the current version, read from a file called $<$ LSEQ $>$, Lander Sequence of Events. Additionally, PREFEP transforms the time format from the bare on-board counter time to one utilizing information about the mission epoch in addition to the counter, sorts the good frames according to Lander Local Time (LLT), and outputs the frames in a Front End Processor (FEP) compatible form. This form is called $<$ MFILE $>$. The time transformation becomes necessary as the original time counter covers only the three month mission time planned in the beginning, after which it resets to zero and starts over. The epoch is an

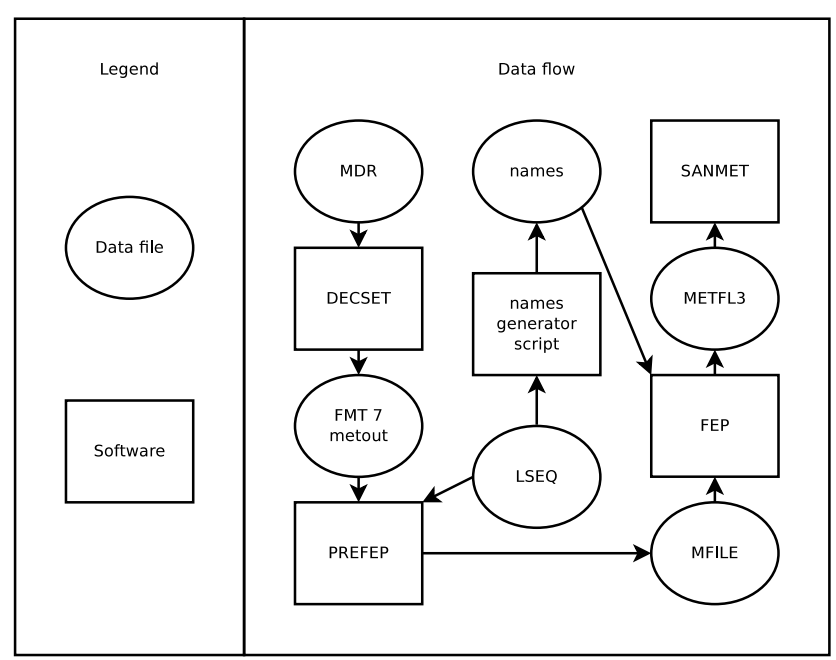

Fig. 1. Flow chart of the analysis package. The explanations of the abbreviations are explained in Sect. 3 .

additional counter, measuring how many times the on-board counter has overflown, i.e. reset to zero. Each "time" information therefore has to be corrected with the related epoch to generate an unambiguous linear time scale across the whole mission. PREFEP general structure and the individual subparts of the program are briefly described in Table 2 .

After or concurrently to PREFEP a $<$ names $>$ generator script is run. This script generates a parameter file called $<$ names $>$ for each sol. Each $<$ names $>$ contains information about various processing variables, data timing, and instrument voltage limits for one sol. The original $<$ names $>$ were created manually and are now unavailable. The variables used are partially based on the few original $<$ names $>$ 
Table 3. Bit error and outlier detection (FEP).

\begin{tabular}{ll}
\hline Name & Description \\
\hline INPUT & Handles parameters and file reads \\
BITDRP & Counts the number of usable samples and check for possible bit drop outs \\
PRTY & Checks for parity errors, and discards frames in which they are found \\
BITER & Calculates the bit error rate by checking the bits of measurements that should be all 1's \\
DPLCT & Searches for duplicate data frames and discards the current one if a duplicate one is found \\
PRSUR & Interpolates pressure values from a pre-calculated table generated from the pressure instrument data \\
ALARM & $\begin{array}{l}\text { Checks that the voltage values fit into the limits set in a LSEQ file, and if not, replaces them with defaults found in the } \\
\text { same file }\end{array}$ \\
REJCT & $\begin{array}{l}\text { Searches for outliers by checking each value against the mean and standard deviation of the rest of the data, and in case a } \\
\text { discrepancy is found, replaces the value by a running mean }\end{array}$ \\
OUTPUT & \begin{tabular}{l} 
Outputs the data \\
\hline
\end{tabular}
\end{tabular}

Table 4. Supporting scientific variable calculation (SANMET general).

\begin{tabular}{ll}
\hline Name & Description \\
\hline DATAB & Covers the parameter, file read and variable initialization part of SANMET \\
BLDVL & Reads various scientific constants from a parameter file \\
MTRIX1 & Changes the coordinate system from horizontal to that of the Viking Lander \\
CMPFCT & Calculates the compressibility factor of the atmosphere based on the pressure and information of its constituents \\
TABLUK & Converts data frame Julian times to $L_{\mathrm{S}}$ and Lander Local Time \\
ANGLS & Calculates sun elevation and its azimuth at the Viking Lander location based on the timing information \\
DATA6 & Converts voltage values to engineering units \\
MOMNT & Calculates the mean, standards deviation and skewness of the data array \\
WNDTMP & Calculates the real scientific temperature and wind values from the raw ones \\
WNDTMP & is explained in a more detail in Table 5 \\
STATIS & Calculates statistical data from the modules \\
OUTPUT & Outputs the data \\
\hline
\end{tabular}

we have, and partially reverse engineered from the program input format requirements.

FEP reads in an $<$ MFILE $>$ and a $<$ names $>$ for each sol, converts the measurement binary data to instrument voltage values, checks if any of the voltage values exceed the set limits and replaces the voltage value with a default value if needed. The voltage data is written in a format called $<$ METFL3 $>$. FEP general structure and the individual subparts of the program are briefly described in Table 3 .

All the actual analysis is done by Meteorology Analysis Program (SANMET). SANMET reads in a $<$ METFL3 $>$ and converts the voltage values of the instruments to engineering variables, which are then used to calculate the scientific variables. The values are written to one-sol files, from which the data is extracted to a final table format for easy usage. SANMET general structure and the individual subparts of the program are briefly described in Table 4. A key subroutine WNDTMP is further described in Table 5. The details of operation can be found in SANMET Program Description Document (Buehler, 1974a) or SANMET Users Guide (Buehler, 1974b).
A sequence number system is used in all the processing phases to preclude the outputs of different runs from overwriting each other. The sequence number system makes it possible to, for example, preserve <MFILE>'s of several PREFEP runs in case the user wants to try different FEP configurations for different PREFEP configurations. As a side effect, it also enables the user to have to run only a part of the processing pipeline in case a software or configuration change is made. As an example, if the user wants to change SANMET run parameters, DECSET, PREFEP, <names $>$ generator script and FEP do not have to be run again, because $<$ METFL3 $>$ files already exist.

\section{Illustration of the new temperature data}

As mentioned in Sect. 1, only the temperature data is discussed here in any detail. Even though the quality control process is still ongoing, we feel confident that the temperature data is fairly accurate. Most of the gaps in the data are caused by a couple of missing raw data tapes. Those 
Table 5. Wind and temperature value calculation (SANMET WNDTMP).

\begin{tabular}{ll}
\hline Name & Description \\
\hline RMARSN & Calculates the distance of Mars from the Sun \\
MSATMP & Calculates the atmospheric temperature \\
CNDCT & Calculates the heat conductivity of the atmosphere \\
VSCSTY & Calculates the viscosity of the atmosphere \\
SIMON & Calculates the wind velocity and direction \\
XFORM & Transforms the wind from the lander coordinates to local horizontal coordinate system \\
EXCES & Adjusts sample pressure based on wind velocity and direction \\
\hline
\end{tabular}

Table 6. Quantitative comparison of PDS and freshly processed (ULT) data.

\begin{tabular}{rrrrrrrrr}
\hline VL & Quant. & Sol range & $\begin{array}{c}\text { Available } \\
\text { sols }\end{array}$ & $\begin{array}{c}\text { Sol } \\
\text { coverage } \\
(\%)\end{array}$ & $\begin{array}{c}\text { Gap } \\
\text { sols }\end{array}$ & $\begin{array}{c}\text { Total } \\
\text { missing } \\
\text { sols }\end{array}$ & $\begin{array}{c}\text { Data } \\
\text { samples }\end{array}$ & $\begin{array}{c}\text { Avg } \\
\text { samples } \\
\text { per sol }\end{array}$ \\
\hline \multicolumn{7}{c}{ OPS DATA (currently at PDS) } \\
\hline 1 & Press. & 2245 & 1547 & 69 & 698 & 698 & 89140 & 57.62 \\
1 & Temp & 350 & 333 & 15 & 17 & 1912 & 8325 & 25 \\
1 & Wind & 350 & 333 & 15 & 17 & 1912 & 8325 & 25 \\
2 & Press. & 1204 & 1001 & 76 & 203 & 323 & 61411 & 61.35 \\
2 & Temp & 1050 & 933 & 70 & 117 & 391 & 23307 & 24.98 \\
2 & Wind & 1050 & 933 & 70 & 117 & 391 & 23307 & 24.98 \\
\hline & & & & ULT DATA & & & \\
\hline 1 & Temp & 2245 & 1561 & 70 & 684 & 684 & 1398709 & 920.2 \\
1 & Wind & 2245 & 1561 & 70 & 684 & 684 & 1398709 & 920.2 \\
2 & Temp & 1281 & 826 & 64 & 455 & 455 & 1141927 & 1418.5 \\
2 & Wind & 1281 & 826 & 64 & 455 & 455 & 1141927 & 1418.5 \\
\hline
\end{tabular}

tapes have been lost for over $25 \mathrm{yr}$, and therefore recovering them seems unlikely. Below follows a description of the data, which is available at present.

Table 6 shows the amount of temperature and wind data produced with ULT and compares it to the amount of data previously published in Planetary Data System (PDS). Although the ULT-produced data contains gaps, it covers a significantly greater portion of VL1 mission's sols than the PDS data, $70 \%$ of the sols compared to $15 \%$. Judging from operational logs and the gaps in PDS pressure data, it seems likely that all the leftover gaps in the ULT data are caused by either operational causes, such as gaps in Deep Space Network coverage or missing raw data tapes, and as such can be assumed to be unrecoverable. However, as PDS has more data for VL2 than ULT data, it is possible that data for some of the gaps still exist in some kind of an alternative or backup data format. In case the reader has any further knowledge of these missing raw data tapes, we kindly ask him or her to contact the authors as soon as possible.

In addition to filling some of the previously void sections of the mission, the amount of data points per sol is much greater in the data produced by ULT. VL1 has on average 36.8 times and VL2 has 56.8 times the data points compared to PDS data. Therefore, whereas the PDS data temporal resolution is one hour, ULT data has mean temporal resolution of $96 \mathrm{~s}$ for VL1 and $63 \mathrm{~s}$ for VL2. The best resolution found for both landers is two seconds, but that was only available for short periods of time. For almost the whole first half of both lander missions (sols 1-847 for VL1, sols 1-695 for VL2), the daytime data resolution is $31 \mathrm{~s}$, and nighttime data resolution is $63 \mathrm{~s}$. After that the data resolution is approximately 9.1 min for VL1 and $11.6 \mathrm{~min}$ for VL2.

For coverage calculations and some figures, the parsed data are binned to 25 evenly distributed bins of equal width (numbered from 0 to 24), and as long as a bin contains at least one data point, it is deemed non-empty. Complete coverage is here defined as some data existing for every $1 / 25$ th of the sol.

For the VL1 ULT, of the 1561 sols that contain some data, 1282 are covered completely. Sols, which have at least one but less than 25 non-empty bins, are called partially covered sols. Partially covered sols have on average $67.6 \%$ (16.9 25 ths) of the sol covered.

For the VL2 ULT of the 826 sols that contain some data, 570 are covered completely. Partially covered sols have on average $68.0 \%$ (17.0 25 ths) of the sol covered. 


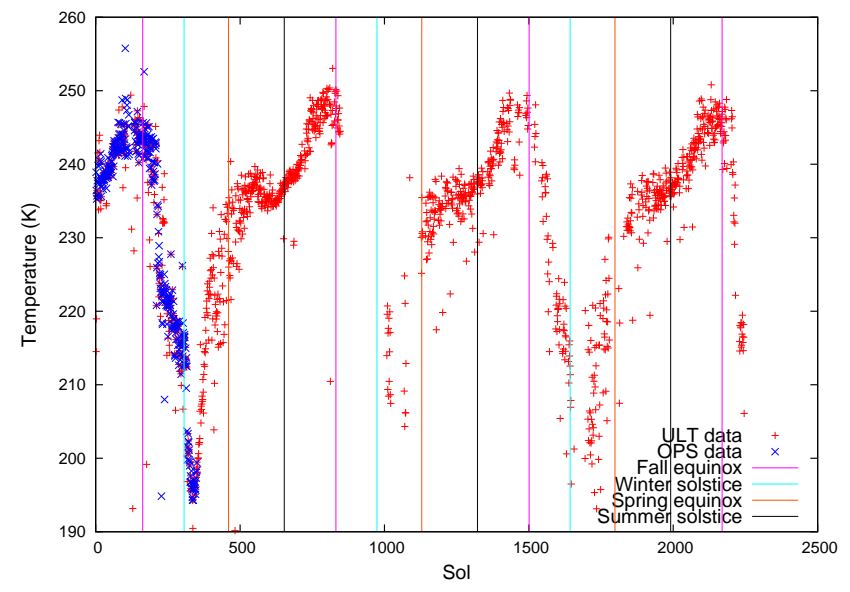

Fig. 2. ULT and OPS temperature data of all the VL1 sols, bin 12.

Freshly produced data by ULT can be illustrated by a few temperature data cases. In Fig. 2 the VL1 mean temperature of (12:00, 13:00 LLT - Lander Local Time) of each sol is shown for sols 1-850 and for both the PDS and ULT data, depicting one full Martian yearly temperature cycle. In addition, equinoxes and solstices are shown with vertical lines. The figure illustrates the fact that the data produced with ULT matches the PDS data fairly well where the latter is available. For some sols there is a difference of several Kelvins, but generally the trends are very similar.

In the following figures, it is good to note that there were several global dust storms during the VL mission and that some of the irregularities in the plots can be explained by them (Tillman et al., 1994). A quantitative description of dust storm effects on the various meteorological quantities will be discussed in an upcoming publication.

The general behavior of both the daily mean and the midday temperature during a Martian year at the VL1 site is as follows. First, the temperature rises from sol 0 until approximately sol 100, when the temperature is at its yearly noon maximum of approximately $245 \mathrm{~K}$. After that, the temperature starts to decrease for 230 sols. The decrease is not linear, but instead consists of two periods of fast decrease and one period of slow decrease. The decrease ends in the yearly minimum temperature of approximately $200 \mathrm{~K}$.

After the minimum, the temperature starts to rise, first rapidly for 70 sols and then slower for 120 sols. Then, at about $550 \mathrm{sol}$, the temperature is at a local maximum. The maximum ends when the temperature decreases approximately $3 \mathrm{~K}$, after which it starts to rise again. The rise is slow for 100 sols, but becomes faster after that. Finally, the yearly maximum is reached on about sol 800 .

Figure 3 shows the VL1 temperature plots of different times of a sol. The noon temperature peaks close to the fall equinox, after which it starts to decrease rapidly. The minimum temperature is achieved approximately 20 sols after the winter solstice. After that, the temperature starts to rise

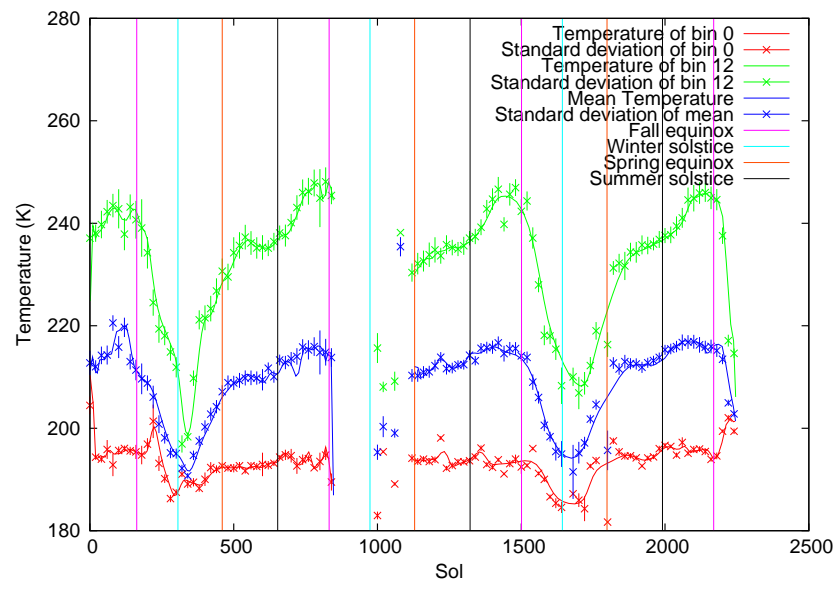

Fig. 3. ULT VL1 noon, midnight and sol mean temperature data of the whole mission.

again in the beginning approximately at the same rate as it decreased during late fall and winter. The rising of the temperature slows down after the vernal equinox and comes to almost a full stop shortly before summer solstice. During the first year, the noon temperature actually seems to decrease a couple of degrees near summer solstice. After the solstice, the temperature starts to rise again until the maximum at fall equinox.

The midnight temperature has a low variability compared to the noon temperature. The midnight minimum temperature is approximately $20^{\circ}$ lower than the midnight maximum temperature. In contrast, the difference between the maximum and the minimum noon temperature is approximately $50^{\circ}$. The sol mean is, by visual inspection, approximately the mean of midnight and noon temperatures. The yearly minima and the maxima of all the temperature curves coincide fairly accurately.

VL2 plots are shown in Figs. 4 and 5. The behavior is similar in the sense that there are clear diurnal and seasonal variations, and that diurnal variation is much greater during summer than during winter. VL2 plot is significantly more symmetrical and smooth. The shoulder during early winter is almost invisible, and the dip around the summer solstice is much smaller. Moreover, the seasonal variation in midnight temperature is much greater.

In the following text the time is given in LLT, which is the local solar time at the location of the lander. LLT runs from 00:00:00 to 24:39:35 LLT, where hours, minutes and seconds are equal to those on Earth, and 00:00:00 LLT is the midnight. In addition, for easier comparison to figures, the time is given in a fraction format in parentheses following the LLT. As an example of the usage, the sol begins at 00:00 LLT (.0) and noon occurs at 12:20 LLT (.5). For simplicity, the LLT is rounded to the nearest minute.

Figure 6 shows a temperature plot of sol 645. During the night, the temperature decreases steadily until 04:56 LLT 


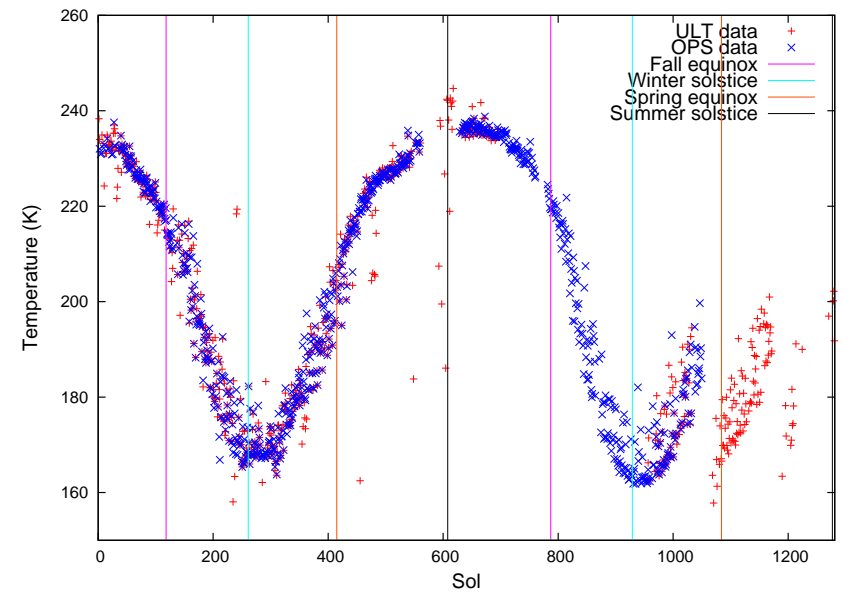

Fig. 4. ULT and OPS temperature data of all the VL2 sols, bin 12.

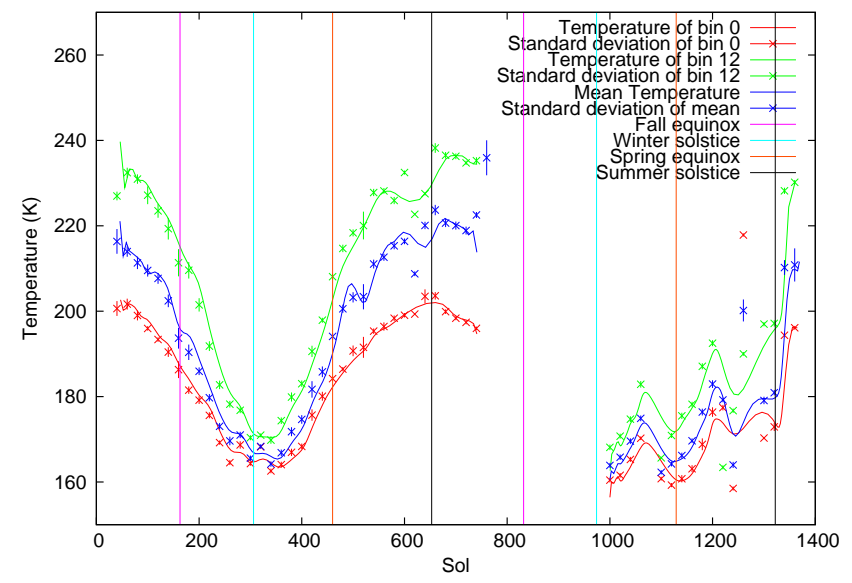

Fig. 5. ULT VL2 noon, midnight and sol mean temperature data of the whole mission.

(.2). After that, the temperature starts to climb with the rate of approximately $7.4 \mathrm{Kh}^{-1}$ until 12:20 LLT (.5). The peak temperature is sustained for $3.5 \mathrm{~h}$, after which the temperature starts to decrease and does so for the rest of the sol.

Figure 7 further illustrates the resolution by showing a 4.93-h (17755 s) period of the sol 645, from 07:24 LLT (.3) to 12:20 LLT (.5). This is the period of the morning when the temperature is rising constantly, but with a large fluctuation lasting for approximately $1 \mathrm{~h}$, shown as a bump in the otherwise quite clean plot.

\section{Summary and discussion}

Viking Lander missions, especially Viking Lander 1 (VL1), are potentially the best source of long-term meteorological data from Mars at least until the year 2018. Unfortunately, the present state is that only a small fraction of the data has been processed and made public. To address this, we have developed a new analysis toolbox, called Updated Linux Toolbox

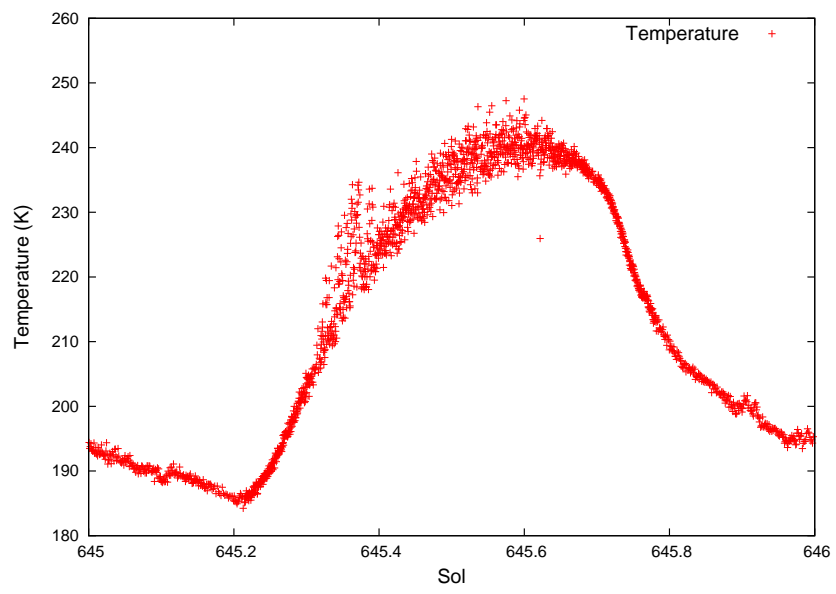

Fig. 6. ULT temperature data of VL1 sol 645.

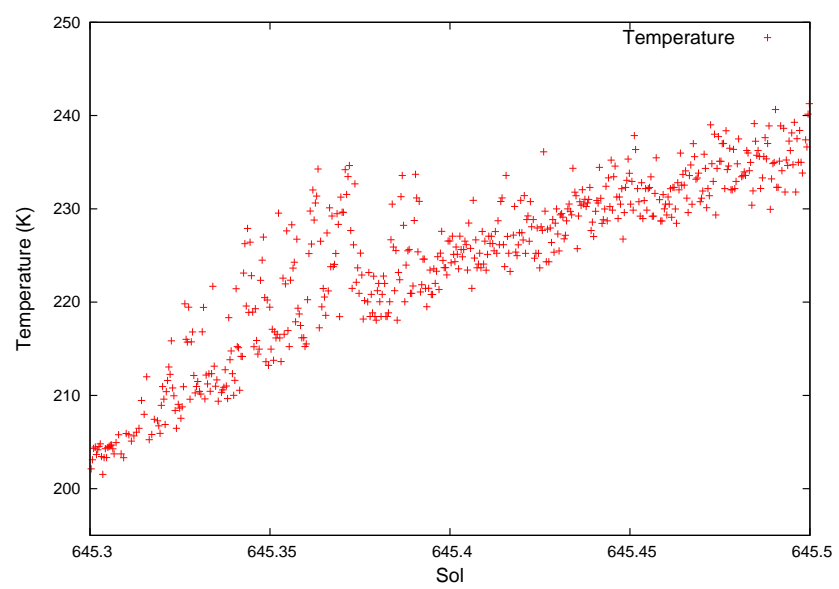

Fig. 7. ULT temperature data of a 4.93-h morning period of VL1 sol 645 .

(ULT). A major part of ULT was developed by using and modifying the old VCF Prime computer software. Additional algorithms and implementation procedures were created by analyzing the limitations of the original VL software and by consulting the vast VL document archives at the VCF. Eventually, the ULT was implemented on a Linux platform.

The current version of ULT provides a straightforward way to analyze the whole data. Running the software does not require any in-depth knowledge of the software or the mission details. The segmented software enables faster processing, because the user does not have to run unaffected parts of the toolbox. For example, when changing the wind calculations, only SANMET has to be run.

ULT currently runs in a local Linux cluster at FMI, but has been tested to run also in a cloud. Other than that, there are no special requirements, though to compile the software one needs a Fortran 90 compiler. The processing time of the whole software chain from raw data to processed meterological data is, for one lander, on the order of one working day, 
i.e. $8 \mathrm{~h}$, on a single $2.66 \mathrm{GHz}$ core. The software does not at present time implement any multi-thread parallelization, but due to the discrete and independent nature of sol-specific analysis it would be suited to parallelization very well. If a faster performance is desired that is an obvious step to perform, in addition to running the software on a faster core. In theory, with a thousand cores, each of them processing data of a single sol, the processing time should be very fast, no more than a few minutes.

The memory consumption of ULT is at most a few hundred MB during processing. With multiple threads the memory consumption would likely increase almost linearly as a function of the number of threads. A complete set of processed data, all phases combined, takes approximately $2.5 \mathrm{~GB}$ of hard disk space. The raw data takes a few hundred MB of hard disk space. The source code of ULT takes a few MB of hard disk space.

The general structure of ULT is the same as in the original analysis software and consists of four core packages that are run linearly. In addition, we developed a new algorithm called $<$ names $>$ generator to enable the analysis of the previously unprocessed VL data.

The first package, DECSET, extracts meteorological data frames from the raw bit stream. The second package, PREFEP, matches the data frames to known measurement times. The $<$ names $>$ generator script generates runparameter files based on PREFEP output. The third core package, FEP, performs bit-level verification for the PREFEP output and additionally replaces data points that are flagged as outliers by pre-determined backup values. The fourth and final package, SANMET, calculates the atmospheric temperature and the wind vector.

ULT and the data produced by it are currently undergoing a validation process. Barring any discoveries of serious problems, we plan to release the data during years 2013-2014 in the PDS. ULT itself, as well as the documentation, will be available via a request.

For temperature and wind data, ULT has 4.7 times as many VL1 data sols and 0.89 times as many VL2 data sols. The amount of data points per data sol compared to Planetary Data System (PDS) data are 36.8 times as much for VL1 and 56.8 times as much for VL2. Judging from operational logs and PDS pressure data, it seems that all the remaining gaps are caused by missing Magnetic Data Record (MDR) tapes or operational causes, and therefore unrecoverable. However, as PDS data has more data sols than ULT data, it is possible that some of the gaps can still be recovered with some kind of an alternative or backup source.
Acknowledgements. The authors are very grateful for the original VCF team, specifically Dr. Neal Johnson, for preserving the data and creating much of the software used here, as well as providing detailed support for our work. We are thankful for the Academy of Finland for their support of the work (grant \#132825). In addition, we want to thank Rami Järvinen, Mark Paton and Visa Hankala for the initial porting and investigative work.

Edited by: L. Vazquez

\section{References}

Buehler, G. D.: SD-37P0011A Viking'75 Project Program Description Document For The Meteorology Analysis Program (SANMET), Project documentation, National Aeronautics and Space Administration (NASA), 1974a.

Buehler, G. D.: SD-37U0011A Viking'75 Project Users Guide For The Meteorology Analysis Program (SANMET), Project documentation, National Aeronautics and Space Administration (NASA), 1974b.

Chamberlain, T. E., Cole, H. L., Dutton, R. G., Greene, G. C., and Tillman, J. E.: Atmospheric Measurements on Mars: The Viking Meteorology Experiment, B. Am. Meteorol. Soc., 57, 1094-1104, 1976.

Golombek, M. P., Anderson, R. C., Barnes, J. R., Bell III, J. F., Bridges, N. T., Britt, D. T., Brückner, J., Cook, R. A., Crisp, D., Crisp, J., Economou, T., Folkner, W. M., Greeley, R., Haberle, R. M., Hargraves, R. B., Harris, J. A., Haldemann, A. F. C., Herkenhoff, K. E., Hviid, S. F., Jaumann, R., Johnson, J. R., Kallemeyn, P. H., Keller, H. U., Kirk , R. L., Knudsen, J. M., Larsen, S., Lemmon, M., Madsen, M. B., Magalhães, J. A., Maki, J. N., Malin, M. C., Manning, R. M., Matijevic, J., Y. McSween Jr. and H. J. Moore and S. L. Murchie and J. R. Murphy and T. Parker, H. J., Rieder, R., Rivellini, T. P., Schofield, J. T., Seiff, A., Singer, R., Smith, P. H., Soderblom, L. A., Spencer, D. A., Stoker, C., Sullivan, R., Thomas, N., Thurman, S. W., Tomasko, M. G., Vaughan, R. M., Wänke, H., Ward, W., and Wilson, G.: Overview of the Mars Pathfinder Mission: Launch through landing, surface operations, data sets, and science results, J. Geophys. Res., 104, 8523-8553, 1999.

Harri, A.-M., Linkin, V., Polkko, J., Marov, M., Pommereau, J.P., Lipatov, A., Siili, T., Manuilov, K., Lebedey, V., Lehto, A., Pellinen, R., Pirjola, R., Carpentier, T., Malique, C., Makarov, V., Khloustova, L., Esposito, L., Maki, J., Lawrence, G., and Lystsev, V.: Meteorological observations on Martian surface : met-packages of Mars-96 Small Stations and Penetrators, Planet. Space Sci., 46, 779-793, doi:10.1016/S0032-0633(98)00012-9, 1998.

Linkin, V., Harri, A.-M., Lipatov, A., Belostotskaja, K., Derbunovich, B., Ekonomov, A., Khloustova, L., Kremnev, R., Makarov, V., Martinov, B., Nenarokov, D., Prostov, M., Pustovalov, A., Shustko, G., Järvinen, I., Kivilinna, H., Korpela, S., Kumpulainen, K., Lehto, A., Pellinen, R., Pirjola, R., Riihelä, P., Salminen, A., Schmidt, W., Siili, T., Blamont, J., Carpentier, T., Debus, A., Hua, C. T., Karczewski, J.-F., Laplace, H., Levacher, P., Lognonné, P., Malique, C., Menvielle, M., Mouli, G., Pommereau, J.-P., Quotb, K., Runavot, J., Vienne, D., Grunthaner, F., Kuhnke, F., Musmann, G., Rieder, R., Wänke, H., Economou, T., Herring, M., Lane, A., and McKay, C. P.: A sophisticated lander 
for scientific exploration of Mars: scientific objectives and implementation of the Mars-96 Small Station, Planet. Space Sci., 46, 717-737, doi:10.1016/S0032-0633(98)00008-7, 1998.

Savijärvi, H., Määttänen, A., Kauhanen, J., and Harri, A.-M.: Mars Pathfinder: New data and new model simulations, Q. J. Roy. Meteorol. Soc., 130, 669-683, doi:10.1256/qj.03.59, 2004.

Savijärvi, H., Crisp, D., and Harri, A.-M.: Effects of $\mathrm{CO}_{2}$ and dust on present-day solar radiation and climate on Mars, Q. J. Roy. Meteorol. Soc., 131, 2907-2922, doi:10.1256/qj.04.09, 2005.

Shotwell, R.: Phoenix - the first Mars Scout mission, Acta Astronaut., 57, 121-134, 2005.

Smith, M. D., Wolff, M. J., Spanovich, N., Ghosh, A., Banfield, D., Christensen, P. R., Landis, G. A., and Squyres, S. W.: One Martian year of atmospheric observations using MER Mini-TES, J. Geophys. Res., 111, E12S13, doi:10.1029/2006JE002770, 2006.

Snyder, C. W.: The Missions of the Viking Orbiters, J. Geophys. Res., 82, 3971-3983, 1977.

Soffen, G. A.: The Viking Project, J. Geophys. Res., 82, 3959-3970, 1977.
Taylor, P. A., Catling, D. C., Daly, M., Dickinson, C. S., Gunnlaugsson, H. P., Harri, A.-M., and Lange, C. F.: Temperature, pressure, and wind instrumentation in the Phoenix meteorological package, J. Geophys. Res.-Planets, 113, E00A10, doi:10.1029/2007JE003015, 2008.

Taylor, P. A., Kahanpää, H., Weng, W., Akingunola, A., Cook, C., Daly, M., Dickinson, C., Harri, A.-M., Hill, D., Hipkin, V., Polkko, J., and Whiteway, J.: On pressure measurement and seasonal pressure variations during the Phoenix mission, J. Geophys. Res.-Planets, 115, E00E15, doi:10.1029/2009JE003422, 2010.

Tillman, J. E., Johnson, N. C., and Smith, R. E.: The Viking Lander Master Data Record: Tape archives, engineering data processing and science data recovery, rev. 2, Project documentation, Department of Atmospheric Sciences, University of Washington, 1988.

Tillman, J. E., Landberg, L., and Larsen, S. E.: The boundary layer of Mars: Fluxes, stability, turbulent spectra, and growth of the mixed layer, J. Atmos. Sci., 51, 1709-1727, 1994.

Williams, D. R.: Viking Project Information, available at: http://nssdc.gsfc.nasa.gov/planetary/viking.html, last access: 30 May 2011. 